\title{
Coffee consumption and NAFLD: a community based study on 1223 subjects
}

\author{
Tilmann Graeter ${ }^{1 \dagger}$, Pia C. Niedermayer ${ }^{2 \dagger}$, Richard A. Mason ${ }^{3}$, Suemeyra Oeztuerk², Mark M. Haenle ${ }^{2}$, \\ Wolfgang Koenig ${ }^{4}$, Bernhard Otto Boehm ${ }^{2,5,6}$, Wolfgang Kratzer ${ }^{2 *}$ and for the EMIL-Study group
}

\begin{abstract}
Background: Objective of the present cross-sectional study was to investigate the impact of caffeine consumption on fatty liver and serum alanine aminotransferase (ALT) concentrations in a random population sample.

Methods: All subjects ( $n=1452 ; 789$ women, 663 men; average age $42.3 \pm 12.8$ years) underwent ultrasonographic examination of the liver and completed a standardized questionnaire regarding personal and lifestyle data, in particular relating to coffee consumption and past medical history. In addition, anthropometric data were documented and laboratory examinations performed. Statistical interpretation of the data was performed descriptively and by means of bivariate and multivariate analysis.

Results: Data of the present study demonstrated a significant association between hepatic steatosis male gender $(p<0.0001)$, advanced age ( $p<0.0001)$ and elevated body-mass index (BMl; $p<0.0001)$. No association between caffeine consumption and fatty liver was identified. An association between caffeine consumption and elevated serum ALT concentrations was not identified.
\end{abstract}

Conclusions: The findings of the present study provide no evidence for an association between caffeine consumption and either the prevalence of hepatic steatosis or serum ALT concentrations.

Keywords: Caffeine, Hepatic steatosis, Fatty liver, Alanine aminotransferase, Population-based cross-sectional study

\section{Background}

Non-alcoholic fatty liver disease (NAFLD) is the most frequent and most important liver disorder worldwide [1]. The increase in overweight, type-2 diabetes mellitus and dyslipidemia has reached epidemic proportions. The development of NAFLD is closely associated with all three of these disorders [2]. The spectrum of NAFLD extends from simple deposits of fat in the liver to nonalcoholic steatohepatitis (NASH), fibrosis and, ultimately, cirrhosis. Histology reveals triglyceride-containing fat vesicles within the hepatic parenchyma that are characteristic of simple fat deposits; NASH is characterized by inflammatory infiltrates and cell destruction [3]. In

\footnotetext{
*Correspondence: wolfgang.kratzer@uniklinik-ulm.de

†Tilmann Graeter and Pia C. Niedermayer contributed equally to this work

2 Department of Internal Medicine I, University Hospital UIm,

Albert-Einstein-Allee 23, 89081 Ulm, Germany

Full list of author information is available at the end of the article
}

cirrhosis, scar tissue replaces the degenerated hepatic parenchyma.

Fatty liver is diagnosed using imaging methods such as ultrasonography (US), and magnetic resonance imaging (MRI). MRI is superior to ultrasound in detecting fatty liver, especially when the degree of hepatic steatosis is mild. Using ultrasound is practical and cost-effective and is associated with a sensitivity of $83 \%$ and specifity of $100 \%$ if at least $30 \%$ of the liver has been affected by fat accumulation and has been shown to be very valuable clinically in assessing the fat content of the liver [4].

Laboratory parameters, such as an elevated transaminase quotient (aspartate transaminase (AST)/alanine transaminase (ALT)) or a depressed adiponectin concentration can provide some assistance in distinguishing steatohepatitis from simple fatty liver [5]. Liver biopsy with histological examination remains, however, the gold standard for diagnostic confirmation $[6,7]$. 
Coffee is one of the most widely consumed beverages worldwide [8]. Beneficial effects of coffee consumption have been demonstrated for a wide variety of disorders, including Parkinson's disease, diabetes mellitus, colorectal carcinoma, coronary artery disease, liver diseases and suicidality $[9,10]$. Coffee consumption appears to even reduce mortality $[9,10]$.

Coffee consumption has its beneficial impact particularly on diseased or pathologically altered hepatic parenchyma. Several studies have shown that coffee consumption is associated with a less pronounced degree of fibrosis or cirrhosis of the liver [11-14], a lower bright liver score (BLS) $[11,15]$ and a lower prevalence and reduced severity of NAFLD [11, 16]. Furthermore, a number of studies have uncovered evidence that coffee consumption appears to associated with a lower concentration of ALT [17-19]. The influence of coffee consumption in hepatitis B patients remains controversial $[20,21]$.

To our knowledge, no studies of the effects of coffee consumption of hepatic steatosis and ALT concentrations in non-selected populations have been published. Objective of the present study was to investigate this potential relationship in a random population-based sample of individuals aged $18-65$ years.

\section{Methods}

\section{Study population/subjects}

The EMIL Study (Echinococcus multilocularis and other Internal medical disorders in Leutkirch) was conducted in 2002 to investigate the respective prevalences of Echinococcus multilocularis infection and other disorders in the southwest German city of Leutkirch [22]. A total of 4000 registered inhabitants between the ages of 10 and 65 years received invitations by mail to participate in a random population-based sample. Of these, 2445 persons provided their written consent and were examined for the study. In order to determine the effects of coffee consumption on hepatic steatosis, certain exclusion criteria were established. Excluded from the study were all minors; all subjects with history of hepatitis B or C, or of hemochromatosis and all subjects reporting alcohol abuse (males, $\geq 40 \mathrm{~g} / \mathrm{d}$; females, $\geq 20 \mathrm{~g} / \mathrm{d}$ ). Also excluded were any subjects with incomplete data sets. The resulting study collective thus included 1452 subjects (Fig. 1).

\section{Quantification of caffeine consumption}

Subjects' consumption of caffeinated beverages such as coffee and black tea was assessed using a standardized questionnaire. Quantification of caffeine intake was based on the following categories: more than once a day, daily, less than weekly, less than monthly, seldom/ rarely.

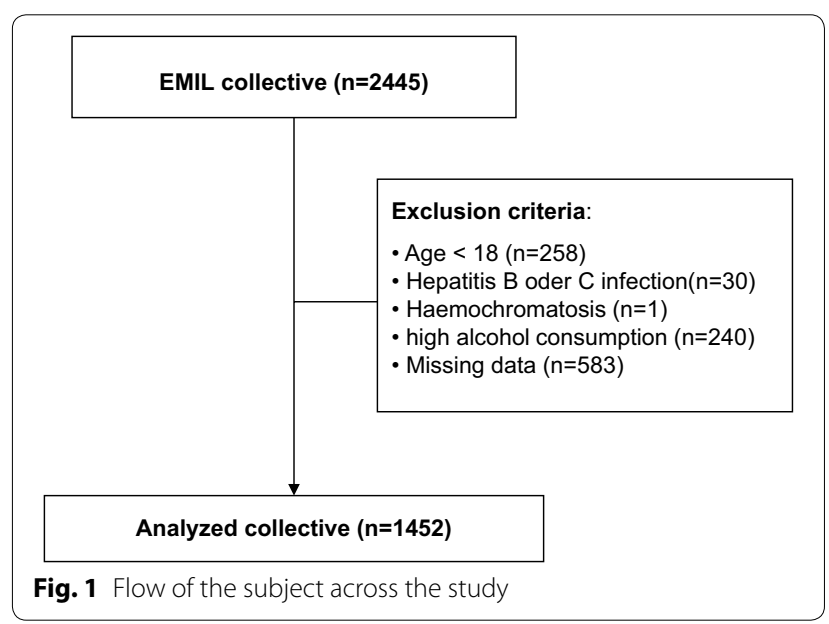

\section{Hepatic steatosis, ultrasonographic criteria}

Four identical HDI 5000 units (ATL Ultrasound, Philips Medical Systems, Bothell, WA, USA) were used. The diagnosis of hepatic steatosis was made according to criteria proposed by Charatcharoenwitthaya based on a comparison of the hepatic and renal parenchyma, taking into consideration the dorsal attenuation, penetration of the diaphragm and ability to assess the liver vessels. The severity of the disease is defined as no steatosis and steatosis grade I, II and III [23, 24]. Due to the small number of cases in group III, these subjects were combined with those in group II for the statistical analysis.

\section{Laboratory tests and procedures}

Laboratory blood testing included analytical clinical chemistry for liver enzymes, lipids and other biochemical values were determined using the Dimension XL unit (Dade Behring Inc., Newark, DE, USA).

\section{Metabolic syndrome: criteria}

Metabolic syndrome was defined according to modified 'US National Cholesterol Education Program Adult Treatment Panel III' criteria, whereby at least three of the following criteria had to be met: Obesity with bodymass index (BMI) over $30 \mathrm{~kg} / \mathrm{m}^{2}$; serum triglyceride (TG) concentration $\geq 1.69 \mathrm{mmol} / \mathrm{l}$; serum high-density lipoprotein (HDL) cholesterol $<1.29 \mathrm{mmol} / \mathrm{l}$ for women and $<1.04 \mathrm{mmol} / \mathrm{l}$ for men; history of hypertension and random blood glucose $>8.88 \mathrm{mmol} / \mathrm{l}$ or confirmed diabetes mellitus [24].

\section{Statistical analysis}

The statistical analysis and calculations were performed with the SAS statistical software program (version 9.2). First, data were analyzed descriptively: for qualitative variables, the relative and absolute frequency were calculated, for quantitative variables the mean and standard 
deviation. In order to detect differences between subjects with and without hepatic steatosis, the Wilcoxon ranksum test was used for continuous variables, while for categorical variables, the Chi-Square test were used. Univariate logistic regression was used to assess the association between caffeine consumption and hepatic steatosis. Multivariate logistic analysis was performed to evaluate the association between hepatic steatosis and caffeine consumption as well as other risk factors like age, BMI, gender, metabolic syndrome and physical activity. To assess the association between elevated ALT concentrations and coffee consumption univarite and multivariate logistic regression analysis was used.

All tests were two-tailed. Statistical significance was set at $\alpha=0.05$. The $P$ value was given to four decimal places, while the odds ratio (OR) and $95 \%$ confidence interval (CI) were given to three decimal places.

\section{Ethical agreement and informed consent}

The study meets the criteria of the revised version of the World Medical Association Declaration of Helsinki (2000) concerning ethical principles for medical research involving human subjects and was approved by the ethics committee of the State Medical Association of BadenWürttemberg. Each study participant provided his or her written consent.

\section{Results}

\section{Study subject demographics}

The data of 1452 subjects [789 women (54.3\%), 663 men $(45.7 \%$ ), average age $42.3 \pm 12.8$ years (range 18-65 years)] were analyzed in the present study. The majority of the subjects were of German nationality $(89.9 \%)$. The mean waist-to-hip ratio (WHR) stood at $0.8 \pm 0.1$, the mean BMI was $25.6 \pm 4.7 \mathrm{~kg} / \mathrm{m}^{2}$. Criteria for metabolic syndrome were met in $5.9 \%$ of subjects. Hepatic steatosis was diagnosed in 381 subjects (26.2 \%) and is more common in men $(34.8 \%, \mathrm{n}=231)$ than in women $(19.0 \%, \mathrm{n}=150)$. The difference in the frequency of hepatic steatosis between men and women was statistically significant $(\mathrm{p}<0.0001)$. Grade I steatosis was identified in 163 subjects (11.2 \%), and grade II/III steatosis was identified in 218 subjects (15.0\%; Fig. 2). Subjects with hepatic steatosis had higher BMI, waist-to-hip ratio (WHR) and transaminase levels (ALT, AST, GGT; Table 1). Elevated ALT concentrations were identified in $198(13.6 \%)$ subjects.

In all, $76.2 \%$ of subjects consumed caffeine at least daily. In our study collective, there was no statistically significant difference between the sexes in terms of caffeine consumption $(\mathrm{p}=0.1748)$. In the univariate analysis, a significant association could be identified between caffeine consumption and hepatic steatosis $(\mathrm{p}=0.0461)$.
Multivariate logistic analysis was performed to evaluate the association between hepatic steatosis and caffeine consumption as well as other risk factors like age, BMI, gender, metabolic syndrome and physical activity. The results show a significant association of heaptic steatosis with gender, age and BMI $(\mathrm{p}<0.0001)$. A relationship between hepatic steatosis and caffeine consumption could not be shown ( $\mathrm{p}=0.8144$, Table 2$)$.

In a further analysis, the impact of coffee on ALT concentrations was assessed. Both in the univariate analysis and after adjusting for gender, age and BMI, there is no significant association between coffee consumption and elevated ALT concentrations ( $\mathrm{p}=0.7495 ; \mathrm{p}=0.7905$; Table 3).

\section{Discussion}

To date to our knowledge, no prospective, sonographic studies of the association between coffee consumption and NAFLD in large population-based collectives have been published. Previous community-based studies have investigated collectives with small numbers of subjects $[13,15]$ or have been retrospective and did not employ diagnostic ultrasonography [16]. Birerdinc et al. retrospectively analyzed 18,550 datasets from the NHANES study. The diagnosis of NAFLD, however, was made exclusively on the basis of elevated transaminase concentrations. Subjects in the NAFLD group consumed significantly less coffee than did the control group ( $p=0.0003$ ) [16]. Studies of elevated ALT concentrations showed significant differences with respect to the populations studied and do not permit any definitive conclusions regarding NAFLD as a cause of the ALT elevation [25]. The findings of this study, therefore, cannot be compared with our findings. Molloy et al. examined a patient collective using both ultrasonography and biopsy, and found that increased coffee consumption was associated with a reduced risk of liver fibrosis in patients with NASH [13]. For ethical reasons, histological diagnosis of NASH cannot be justified in a population-based study; hence, these data also cannot be directly compared with our own.

In a case-control study of a collective of 130 individuals (with and without hepatic steatosis in $n=73$ and $\mathrm{n}=57$ subjects, respectively), Gutiérrez-Grobe et al. made the diagnosis of NAFLD solely on the basis of ultrasonographic criteria. As in our study, the severity of hepatic steatosis was divided into four grades. The findings of the study failed to demonstrate an unequivocal anti-oxidant effect of coffee though patients with highergrade steatosis drank more coffee than did those with less severe steatosis [26].

In a study of 157 patients and 153 controls, Catalano et al. made the diagnosis of hepatic steatosis using 


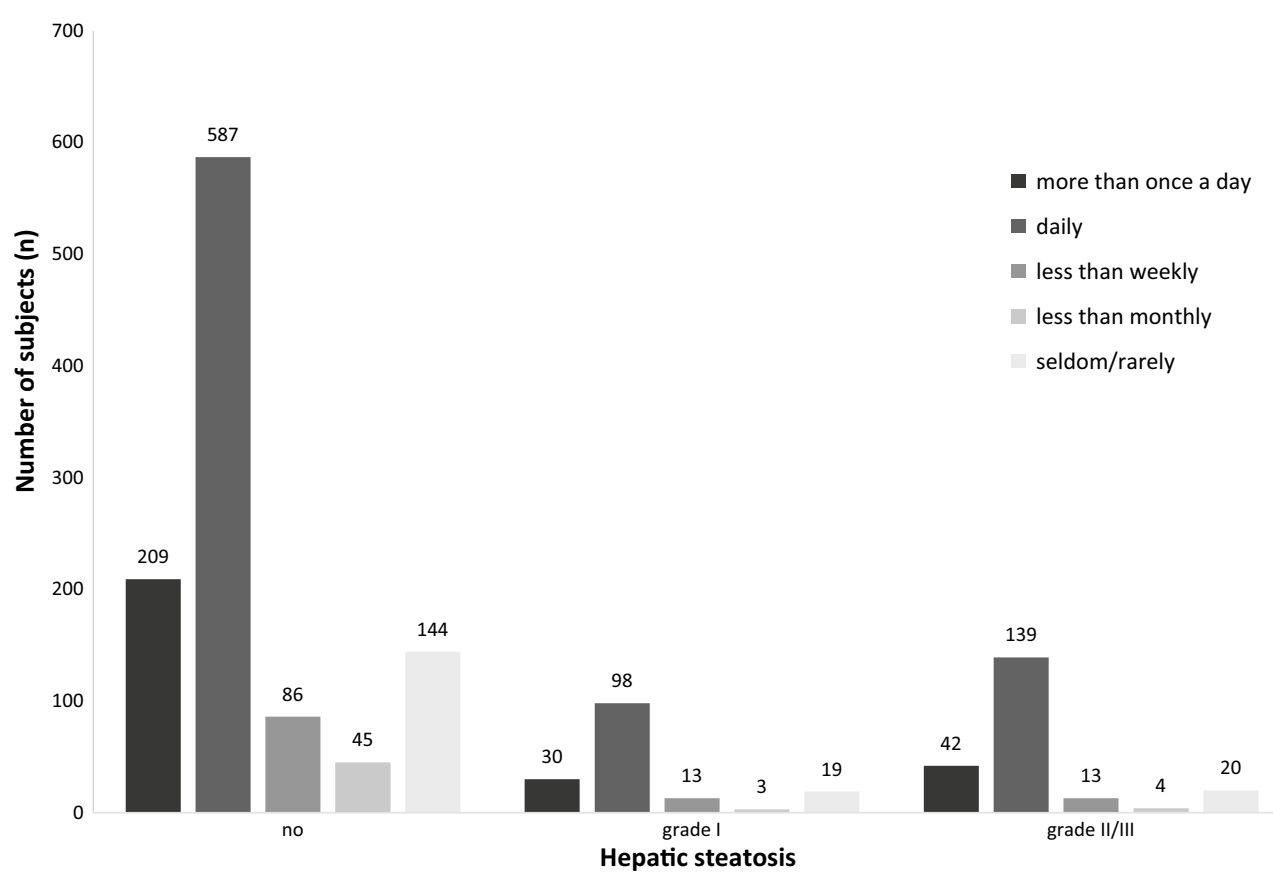

Fig. 2 Caffeine consumption in relation to the severity of hepatic steatosis

Table 1 Demographics and other characteristics of subjects with and without hepatic steatosis

\begin{tabular}{|c|c|c|c|c|}
\hline & $\begin{array}{l}\text { Hepatic steatosis not diagnosed }(n=1071, \\
\text { mean } \pm \text { STD) }\end{array}$ & $\begin{array}{l}\text { Hepatic steatosis diagnosed }(n=381 \text {, } \\
\text { mean } \pm \text { STD) }\end{array}$ & Total & $p$ value \\
\hline \multicolumn{5}{|l|}{ Gender, n (\%) } \\
\hline Female & $639(59.7 \%)$ & 150 (39.4 \%) & $789(54.3 \%)$ & $<0.0001$ \\
\hline Male & $432(40.3 \%)$ & $231(60.6 \%)$ & $663(45.7 \%)$ & \\
\hline Age & $39.9 \pm 12.4$ & $49.0 \pm 11.4$ & $42.3 \pm 12.8$ & $<0.0001$ \\
\hline BMI & $24.1 \pm 3.8$ & $29.8 \pm 4.7$ & $25.6 \pm 4.7$ & $<0.0001$ \\
\hline Waist/hip-ratio & $0.8 \pm 0.1$ & $0.9 \pm 0.1$ & $0.8 \pm 0.1$ & $<0.0001$ \\
\hline \multicolumn{5}{|l|}{ Coffee consumption, n (\%) } \\
\hline More than once a day & $209(19.5 \%)$ & $72(18.9 \%)$ & $281(19.4 \%)$ & \\
\hline Daily & $587(54.8 \%)$ & 237 (62.2 \%) & $824(56.8 \%)$ & \\
\hline Less than weekly & $86(8.0 \%)$ & $26(6.8 \%)$ & $112(7.7 \%)$ & \\
\hline Less than monthly & $45(4.2 \%)$ & $7(1.8 \%)$ & $52(3.6 \%)$ & 0.0408 \\
\hline Seldom/rarely & $144(13.5 \%)$ & $39(10.2 \%)$ & $183(12.6 \%)$ & \\
\hline ALT & $13.3 \pm 5.6$ & $20.0 \pm 10.6$ & $15.1 \pm 7.8$ & $<0.0001$ \\
\hline AST & $8.9 \pm 2.4$ & $11.0 \pm 5.1$ & $9.5 \pm 3.5$ & $<0.0001$ \\
\hline GGT & $11.0 \pm 11.0$ & $19.1 \pm 20.3$ & $13.2 \pm 14.5$ & $<0.0001$ \\
\hline \multicolumn{5}{|l|}{ Metabolic syndrome, n (\%) } \\
\hline No & 1050 (98.0 \%) & 317 (83.2 \%) & 1367 (94.2 \%) & $<0.0001$ \\
\hline Yes & $21(2.0 \%)$ & $64(16.8 \%)$ & $85(5.9 \%)$ & \\
\hline
\end{tabular}

Significant $p$ values are in italics

STD standard deviation, BMI body-mass index, ALT alanine transaminase, AST aspartate transaminase, GGT gamma-glutamyl transferase

different exclusion criteria. Coffee consumption was monitored over a follow-up period of 6 months. The authors thus ascribe a possible protective effect to elevated coffee consumption. Their data, however, were derived from a highly selected collective drawn from ambulatory patients of a gastroenterological practice and 
Table 2 Logistic regression analysis to assess the association between hepatic steatosis and caffee consumption and other influencing factors

\begin{tabular}{lll}
\hline & Odds ratio $\mathbf{9 5}$ \% Cl) & $p$ value \\
\hline $\begin{array}{l}\text { Gender } \\
\text { Female }\end{array}$ & Ref. & $<0.0001$ \\
Male & $2.775(2.047-3.764)$ & \\
Age & $1.053(1.039-1.066)$ & $<0.0001$ \\
BMl & $1.324(1.271-1.380)$ & $<0.0001$ \\
Metabolic syndrome & & \\
No & Ref. & Ref. \\
Yes & $0.906(0.501-1.640)$ & 0.0668 \\
Coffee consumption & & \\
More than once a day & $0.771(0.443-1.343)$ & \\
Daily & $0.809(0.496-1.318)$ & \\
Less than weekly & $0.749(0.368-1.526)$ & \\
Less than monthly & $0.558(0.190-1.638)$ & \\
Seldom/rarely & Ref. & \\
Physical activity & & \\
No & Ref. & \\
0-2 h/week & $1.021(0.719-1.449)$ & \\
2-4 h/week & $0.931(0.612-1.419)$ & \\
$4-10$ h/week & $0.937(0.548-1.603)$ & \\
$>10$ h/week & $0.581(0.169-2.001)$ & \\
\hline
\end{tabular}

Significant $p$ values are in italics

$\mathrm{Cl}$ confidence interval, Ref. reference group

Table 3 Regression analysis to assess the association between coffee consumption and elevated alanine transaminase levels (ALT)

\begin{tabular}{lll}
\hline Coffee consumption & Odds ratio $\mathbf{9 5} \% \mathbf{C l})$ & p value \\
\hline Univariate & & \\
More than once a day & $1.097(0.617-1.953)$ & \\
Daily & $1.302(0.794-2.135)$ & \\
Less than weekly & $1.380(0.694-2.746)$ & 0.7495 \\
Less than monthly & $1.006(0.384-2.640)$ & \\
Seldom/rarely & Ref. & \\
After adjusting for age, gender and BMI & 0.7905 \\
More than once a day & $0.773(0.447-1.339)$ & \\
Daily & $0.806(0.497-1.308)$ & \\
Less than weekly & $0.722(0.355-1.467)$ & \\
Less than monthly & $0.554(0.190-1.613)$ & \\
Seldom/rarely & Ref.
\end{tabular}

$\mathrm{Cl}$ confidence interval, Ref. reference group

outpatient clinic and cannot be applied to the general population [15].

In our study population, elevated ALT concentrations were identified in $13.6 \%$ of subjects. This corresponds with the finding of another cross-sectional study from
China (10.8\%) [25]. Studies published to date have shown an inverse relationship between coffee consumption and serum ALT concentrations. The findings of our population-based study failed to demonstrate a corresponding association between caffeine consumption and serum ALT concentrations. It is to be noted that, in the other studies, the diagnosis of NAFLD was not based on imaging of the liver; furthermore, some of these studies were performed in highly selected populations $[19,27]$.

The present study does have some important limitations. Due to the choice of study design, we were able to investigate associations but not causalities. The diagnosis of hepatic steatosis was made on the basis of ultrasonographic criteria. Liver biopsy, the current gold standard for confirming a diagnosis of NAFLD, was not performed. Furthermore, data on caffeine consumption were obtained only on one occasion. In addition, the dual effect of coffee consumption was not investigated further.

\section{Authors' contributions}

WK, WK and BOB designed the study, took part in the organization of the study and was drafting the manuscript. MM was also part of the study organization team, took part in the data acquisition and interpretation. SO was responsible for data analysis and statistics. TG and RAM drafted the manuscript. PCN and WK wrote the article. All authors read and approved the final manuscript.

\section{Author details}

${ }^{1}$ Department of Diagnostic and Interventional Radiology, University Hospital Ulm, Albert-Einstein-Allee 23, 89081 UIm, Germany. ${ }^{2}$ Department of Internal Medicine I, University Hospital Ulm, Albert-Einstein-Allee 23, 89081 Ulm, Germany. ${ }^{3}$ Louis Stokes Clevel and Department of Veterans Affairs Medical Center, 10700 East Boulevard, Cleveland, OH 44106, USA. ${ }^{4}$ Department of Internal Medicine II, University Hospital Ulm, Albert-Einstein-Allee 23, 89081 Ulm, Germany. ${ }^{5}$ LKC School of Medicine, Nanyang Technological University, Singapore, Singapore. ${ }^{6}$ Imperial College London, London, UK.

\section{Acknowledgements}

This article is based on the doctoral thesis of Pia C. Niedermayer, University Ulm.

The study was initiated be the government of the state of Baden-Württemberg, Germany. Financial support was provided through the Baden-Württemberg state health office of the District Government of Stuttgart, Germany as well as the regional health office of Ravensburg, Germany. Further support was provided by the administration of the city of Leutkirch, Germany. Vials for blood samples and part of the laboratory testing were supplied by Sarstedt AG \& Co, Nürnbrecht, Germany. BOB is supported by the German Research Council (GRK 1041).

The authors wish to express their gratitude to Mr. Walter Feucht of the ULDO Backwaren Company, Neu-Ulm, Germany for his support of this study. Members of the EMIL-Study-Group (Echinococcus Multilocularis and Internal Diseases in Leutkirch): Adler G, Armsen A, Banzhaf H-M, Bauerdick M, Bertling U, Boehm BO, Brandner BO, Brockmann SO, Deckert M, Dingler C, Eggink S, Fuchs M, Gaus W, Goussis H, Gruenert A, Haenle MM, Hampl W, Haug C, Hay B, Huetter M-L, Imhof A, Kern P, Kimmig P, Kirch A, Klass D, Koenig W, Kratzer W, Kron M, Manfras B, Meitinger K, Mertens T, Oehme R, Pfaff G, Piechotowsk I, Reuter S, Romig T, von Schmiesing AFA, Steinbach G, Tourbier M, Voegtle A, Walcher T, WolffS.

\section{Competing interests}

The authors declare that they have no competing interests. 
Received: 30 June 2015 Accepted: 28 October 2015

Published online: 03 November 2015

\section{References}

1. Sanyal AJ. NASH: a global health problem. Hepatol Res. 2011;41:670-4

2. Ballestri S, Lonardo A, Bonapace S, et al. Risk of cardiovascular, cardiac and arrhythmic complications in patients with non-alcoholic fatty liver disease. World J Gastroenterol. 2014;20:1724-45.

3. Takaki A, Kawai D, Yamamoto K. Molecular mechanisms and new treatment strategies for Non-Alcoholic Steatohepatitis (NASH). Int J Mol Sci. 2014;15:7352-79.

4. Qayyum A, Chen DM, Breiman RS, Westphalen AC, Yeh BM, Jones KD, Lu Y, Coakley FV, Callen PW. Evaluation of diffuse liver steatosis by ultrasound, computed tomography, and magnetic resonance imaging: which modality is best? Clin Imaging. 2009;33:110-5.

5. Torres DM, Williams CD, Harrison SA. Features, diagnosis, and treatment of nonalcoholic fatty liver disease. Clin Gastroenterol Hepatol. 2012;10:837-58

6. Torres DM, Harrison SA. Diagnosis and therapy of nonalcoholic steatohepatitis. Gastroenterology. 2008;134:1682-98.

7. Grandison GA, Angulo P. Can NASH be diagnosed, graded, and staged noninvasively? Clin Liver Dis. 2012;16:567-85.

8. Saab S, Mallam D, Cox GA 2nd, et al. Impact of coffee on liver diseases: a systematic review. Liver Int. 2014:34:495-504.

9. Cano-Marquina A, Tarín JJ, Cano A. The impact of coffee on health. Maturitas. 2013;75:7-21.

10. Higdon JV, Frei B. Coffee and health: a review of recent human research. Crit Rev Food Sci Nutr. 2006:46:101-23.

11. Chen S, Teoh NC, Chitturi S, et al. Coffee and non-alcoholic fatty liver disease: brewing evidence for hepatoprotection? J Gastroenterol Hepatol. 2014;29:435-41.

12. Modi AA, Feld JJ, Park Y, et al. Increased caffeine consumption is associated with reduced hepatic fibrosis. Hepatology. 2010;51:201-9.

13. Molloy JW, Calcagno CJ, Williams CD. Association of coffee and caffeine consumption with fatty liver disease, nonalcoholic steatohepatitis, and degree of hepatic fibrosis. Hepatology. 2012;55:429-36.
14. Bambha K, Wilson LA, Unalp A, et al. Coffee consumption in NAFLD patients with lower insulin resistance is associated with lower risk of severe fibrosis. Liver Int. 2014;34:1250-8.

15. Catalano D, Martines GF, Tonzuso A, et al. Protective role of coffee in nonalcoholic fatty liver disease (NAFLD). Dig Dis Sci. 2010;55:3200-6.

16. Birerdinc A, Stepanova M, Pawloski L, et al. Caffeine is protective in patients with non-alcoholic fatty liver disease. Aliment Pharmacol Ther. 2012;35:76-82.

17. Jang ES, Jeong SH, Hwang SH, et al. Effects of coffee, smoking, and alcohol on liver function tests: a comprehensive cross-sectional study. BMC Gastroenterol. 2012;12:145.

18. Muriel P, Arauz J. Coffee and liver diseases. Fitoterapia. 2010;81:297-305.

19. Ruhl CE, Everhart JE. Coffee and caffeine consumption reduce the risk of elevated serum alanin aminotransferase activity in the United States. Gastroenterology. 2005;128:24-32.

20. Ong A, Wong VW, Wong GL, Chan HL. The effect of caffeine and alcohol consumption on liver fibrosis - a study of 1045 Asian hepatitis B patients using transient elastography. Liver Int. 2011;31:1047-53.

21. Jang ES, Jeong SH, Lee $\mathrm{SH}$, et al. The effect of coffee consumption on the development of hepatocellular carcinoma in hepatitis B virus endemic area. Liver Int. 2013;33:1092-9.

22. Haenle MM, Brockmann SO, Kron M, et al. Overweight, physical activity, tobacco and alcohol consumption in a cross-sectional random sample of German adults. BMC Public Health. 2006;6:233.

23. Charatcharoenwitthaya $P$, Lindor KD. Role of radiologic modalities in the management of non-alcoholic steatohepatitis. Clin Liver Dis. 2007;11:37-54.

24. Kratzer W, Akinli AS, Bommer M, et al. Prevalence and risk factors of focal sparing in hepatic steatosis. Ultraschall Med. 2010;31:37-42.

25. Zhang $H$, Jiang $Y F, H e S M$, et al. Etiology and prevalence of abnormal serum alanine aminotransferase levels in a general population in Northeast China. Chin Med J (Engl). 2011;124:2661-8.

26. Gutiérrez-Grobe Y, Chávez-Tapia N, Sánchez-Valle V, et al. High coffee intake is associated with lower grade nonalcoholic fatty liver disease: the role of peripheral antioxidant activity. Ann Hepatol. 2012;11:350-5.

27. Yamashita K, Yatsuya H, Muramatsu T, et al. Association of coffee consumption with serum adiponectin, leptin, inflammation and metabolic markers in Japanese workers: a cross-sectional study. Nutr Diabetes. 2012;2:e33

\section{Submit your next manuscript to BioMed Central and take full advantage of:}

- Convenient online submission

- Thorough peer review

- No space constraints or color figure charges

- Immediate publication on acceptance

- Inclusion in PubMed, CAS, Scopus and Google Scholar

- Research which is freely available for redistribution

Submit your manuscript at

www.biomedcentral.com/submit

C Biomed Central 